\title{
Corrigendum: Lateral Lymph Node Metastases in T1a Papillary Thyroid Carcinoma: Stratification by Tumor Location and Size
}

\begin{abstract}
Xiaojun Zhang ${ }^{1 \dagger}$, Wenkuan Chen ${ }^{2 \dagger}$, Qigen Fang ${ }^{1}$, Jie Fan ${ }^{1}$, Lu Feng ${ }^{1}$, Lanwei Guo ${ }^{3}$, Shanting Liu ${ }^{1}$, Hong $\mathrm{Ge}^{4 *}$ and Wei Du ${ }^{1 *}$

${ }^{1}$ Department of Head Neck and Thyroid Surgery, The Affiliated Cancer Hospital of Zhengzhou University, Henan Cancer Hospital, Zhengzhou, Henan, China, ${ }^{2}$ Department of Head and Neck Surgery, State Key Laboratory of Oncology in South China, Collaborative Innovation Center for Cancer Medicine, Sun Yat-sen University Cancer Center, Guangzhou, China, ${ }^{3}$ Office for Cancer Control and Research, Henan Cancer Hospital, The Affiliated Cancer Hospital of Zhengzhou University, Zhengzhou, China, 4 Department of Radiation Oncology, The Affiliated Cancer Hospital of Zhengzhou University, Henan Cancer Hospital, Zhengzhou, Henan, China
\end{abstract}

Keywords: papillary thyroid carcinoma, lymph node metastases, upper lobe, tumor location, tumor size

\section{OPEN ACCESS}

Approved by:

Frontiers Editorial Office,

Frontiers Media SA, Switzerland

${ }^{*}$ Correspondence:

Wei Du

duweiti@126.com

Hong Ge

gehong616@126.com

${ }^{\dagger}$ These authors have contributed equally to this work and

share first authorship

Specialty section:

This article was submitted to

Thyroid Endocrinology,

a section of the journal

Frontiers in Endocrinology

Received: 11 January 2022 Accepted: 12 January 2022

Published: 01 February 2022

Citation:

Zhang X, Chen W, Fang Q, Fan J, Feng L, Guo L, Liu S, Ge H and Du W (2022) Corrigendum: Lateral Lymph

Node Metastases in T1a Papillary Thyroid Carcinoma: Stratification by

Tumor Location and Size.

Front. Endocrinol. 13:852751. doi: 10.3389/fendo.2022.852751

\section{A Corrigendum on}

Lateral Lymph Node Metastases in T1a Papillary Thyroid Carcinoma: Stratification by Tumor Location and Size

By Zhang X, Chen W, Fang Q, Fan J, Feng L, Guo L, Liu S, Ge H and Du W (2021) Front. Endocrinol. 12:716082. doi: 10.3389/fendo.2021.716082

In the originally published article, affiliations 1 and 4 were presented incorrectly.

Affiliation 1 was presented as "Department of Head Neck and Thyroid Surgery, Henan Cancer Hospital, The Affiliated Cancer Hospital of Zhengzhou University, Zhengzhou, Henan, China"; it should be "Department of Head Neck and Thyroid Surgery, The Affiliated Cancer Hospital of Zhengzhou University, Henan Cancer Hospital, Zhengzhou, Henan, China"

Affiliation 4 was presented as "Department of Radiation Oncology, Henan Cancer Hospital, The Affiliated Cancer Hospital of Zhengzhou University, Zhengzhou, Henan, China"; it should be

"Department of Radiation Oncology, The Affiliated Cancer Hospital of Zhengzhou University, Henan Cancer Hospital, Zhengzhou, Henan, China”.

The authors apologize for this error and state that this does not change the scientific conclusions of the article in any way. The original article has been updated.

Publisher's Note: All claims expressed in this article are solely those of the authors and do not necessarily represent those of their affiliated organizations, or those of the publisher, the editors and the reviewers. Any product that may be evaluated in this article, or claim that may be made by its manufacturer, is not guaranteed or endorsed by the publisher.

Copyright $\odot 2022$ Zhang, Chen, Fang, Fan, Feng, Guo, Liu, Ge and Du. This is an open-access article distributed under the terms of the Creative Commons Attribution License (CC BY). The use, distribution or reproduction in other forums is permitted, provided the original author(s) and the copyright owner(s) are credited and that the original publication in this journal is cited, in accordance with accepted academic practice. No use, distribution or reproduction is permitted which does not comply with these terms. 